\title{
A Computational Analysis on Nicotine Withdrawal and Cardiac Arrhythmia and Their Effects on COVID-19
}

\author{
Ayaan Haque ${ }^{1}$, Aryaman Kukal ${ }^{1}$, Yamuna Rao ${ }^{1}$, David Cho', and Keshav Rao' \\ ${ }^{1}$ American High School, Fremont, CA, USA
}

\section{$\underline{\text { ABSTRACT }}$}

Certain chronic conditions are becoming increasingly ignored due to the magnitude of COVID-19. However, continued research on these conditions, such as nicotine addiction and heart disease, and how they relate to COVID-19 will help researchers assess case severity more adequately. It has been commonly asserted that smoking cessation will reduce COVID-19 mortality rates for smokers due to how it damages the lungs. However, smoking cessation results in nicotine withdrawal, which is proven to cause side effects that could conversely increase the severity of COVID19. This study aims to determine how nicotine withdrawal increases the probability of developing cardiac arrhythmia, and how that relationship increases COVID-19 mortality rates. To conduct our study, we compiled datasets from various health organizations, including the $\mathrm{CDC}$ and $\mathrm{WHO}$, to find correlations between these three conditions. We first investigated the complications that result from nicotine withdrawal and how they may develop into cardiac arrhythmia. Following initial inquiries, we then calculated how cardiac arrhythmia increases COVID-19 mortality rates. To validate our conclusions, we compared smoking cessation rates with COVID-19 mortality rates in different states to isolate direct correlation between the two conditions. We determined that nicotine withdrawal has a $32.7 \%$ probability of causing cardiac arrhythmia, and that cardiac arrhythmia increases the chance of death from COVID-19 by $14.7 \%$. Ultimately, we found that nicotine withdrawal increases the COVID- 19 mortality rate by $4.8 \%$ compared to the general population, a significant and concerning finding.

\section{Introduction}

COVID-19, otherwise known as the coronavirus, was classified as severe acute respiratory syndrome coronavirus 2 by the WHO. It was first identified in Wuhan, China in December of 2019 and has now spread to almost every country. As of July 18, 2020, more than 14.1 million cases of COVID-19 have been reported, resulting in more than 598,000 deaths, but these numbers are increasing rapidly (Redaction, 2020). COVID-19 has overwhelmed the medical field, and as a result, less research is being done on other prevalent medical issues.

A more chronic issue in American society has been smoking tobacco products, primarily cigarettes. Approximately 34.2 million Americans are reported to have taken the drug (CDC, 2019). Smoking cigarettes can potentially cause long-term diseases such as an increased risk of stroke, brain damage, lung cancer, high blood pressure, and heart disease (Mjøs, 1988). Smoking results in more than 480,000 deaths per year in the US and smokers die around 10 years earlier than their non-smoking counterparts (KFF, 2018). Quitting smoking is difficult because of the highly addictive chemical nicotine. Nicotine is also found in vaping products, which are common alternatives to cigarettes and are prominently consumed by adolescents. Nicotine addiction can be especially harmful to the heart, as it can cause an increase in blood pressure, heart rate, flow of blood to the heart, and the narrowing of arteries. The side effects of nicotine withdrawal are most prevalent within the first 3 - 4 weeks of withdrawal, and is considered to be the biggest hurdle smokers face when trying to quit. Other studies have concluded that smoking cessation can reduce a patient's chance of death from COVID-19 and boost health outcomes (KFF, 2018). However, the side effects of nicotine withdrawal are often unobserved in these studies. In this paper, we aim to find a relationship between smoking 
cessation and COVID-19. We use "smoking cessation" interchangeably with "nicotine withdrawal", as nicotine withdrawal occurs when a person stops smoking since almost all cigarettes contain nicotine (Wadgave, \& Nages, 2016).

Another chronic group of conditions in the US is heart diseases. More specifically, we studied a specific heart disease called cardiac arrhythmia. Cardiac arrhythmia is a prevalent condition that occurs in every 1 out of 18 Americans (NCHS, 2020). It takes place when electrical impulses in the heart are not coordinated properly. The most common symptom of cardiac arrhythmia is palpitations, or a short-lived arrhythmia (HHS, 2013). Palpitations are generally negligible, but frequent palpitations can be of higher concern. These palpitations are also a common symptom of nicotine withdrawal. Although palpitations may resolve themselves within 3 - 4 weeks after a person quits nicotine, the symptoms and health concerns associated with these palpitations can result in severe health outcomes (Brugada, Gürsoy, Brugada, \& Andries, 1993). Our research expands on cardiac arrhythmia and determines how it correlates with COVID-19 death rates.

Although researchers and health officials have come a long way in determining the symptoms and possible causes of higher COVID-19 mortality rates amongst different health demographics, there is still an overwhelming amount of research that needs to be conducted. To find relationships between nicotine withdrawal, cardiac arrhythmia, and COVID-19, we analyzed several datasets to uncover trends and correlations. With access to public journals and datasets, we utilized computational and statistical analytics to conduct our research. We began by finding individual correlations between two complications, and at each step, we validated and confirmed our results. For validation of our findings, we compared the smoking cessation rates and COVID-19 death rates across the US to find a direct correlation between the two conditions.

This paper begins by reviewing our methods for evaluating correlations and rates between factors and diseases. This includes an in-depth explanation of each step of our research, why we took such steps, and what tools were used in the process. We then present our results and explain why our findings are statistically accurate. We provide calculations and visualizations for our compiled results and present a validation study. Finally, we present our results and discuss the significance and implications they hold, as well as any potential shortcomings and future expansion of our research.

\section{Methods}

\section{Data Compilation and Tools}

Our main source of data was the CDC data archives. These datasets were preferable because they were collected by trusted sources under the United States HHS. Additionally, we collected data from various medical archives and journals (HHS, 2013; KFF, 2018; Roser, Ritchie, Ortiz-Ospina, \& Joe Hasell, 2020) after reviewing their results and sources to record and compute specific rates and statistics. Many of the datasets were provided as supplemental material in the journals for open-source use.

To conduct our study, we used various open-source data science tools. We primarily utilized Jupyter Notebook, a python-based data science software, to analyze datasets and create figures. The main python library we used was Pandas, a data analysis tool that allowed us to read and visualize datasets. Along with Pandas, we utilized Matplotlib, Sci-Kit, and Seaborn to create statistical models and graphs.

\section{Data Compilation and Tools}

One extremely common symptom of withdrawal are palpitations. While palpitations are a legitimate symptom of nicotine withdrawal, they usually last for 3 - 4 weeks after initial cessation (Brugada, Gürsoy, Brugada, \& Andries, 1993). However, this is enough time for the side effects to proliferate. For our research, the clinical trials we analyzed on nicotine withdrawal needed to provide statistics and results on how often patients developed cardiac arrhythmia. 
We analyzed a study conducted by the NCBI which recorded the side effects of patients who underwent nicotine replacement therapy, including the number of cigarettes they smoked per day, the number of years they smoked, their pre-existing conditions, and their reported cardiovascular outcomes as a result of the experiment (Mills, Thorlund, Eapen, Wu, \& Prochaska, 2014).

\section{Development of Heart Complications}

To calculate a death rate for patients with cardiac arrhythmia and COVID-19, we analyzed a dataset from the CDC which included underlying health conditions for patients with COVID-19 across the nation (HHS, 2013). The dataset included death counts for patients with COVID-19 and cardiac arrhythmia in each state and was categorized by age group. We calculated a nationwide death count for patients with both conditions by totalling the death counts across age groups and states.

Additionally, the total case count of patients with both cardiac arrhythmia and COVID-19 was needed to calculate a mortality rate. Unfortunately, the datasets which were publicly available contained only information regarding mortalities, not total cases. As a result, we had to accurately estimate how many patients in the US had both diseases. We utilized various statistical methods for estimation. The most statistically sound method was to assume that the percent of patients with cardiac arrhythmia in the US would be identical to the percentage of patients with COVID-19 and cardiac arrhythmia in the US. This is because everyone should have the same chance of getting COVID-19, since it is a disease that is contracted, not developed. Using this percentage, we were able to get a raw case count and then calculated a death rate. With this death rate, we compared it to individual death rates of COVID19 and cardiac arrhythmia to evaluate changes.

\section{Development of Heart Complications}

It was crucial to prove an overarching correlation showing that an increase in smoking cessation rates leads to an increase in COVID-19 mortality rates, regardless of potential palpitations or cardiac arrhythmia. We looked at specific trends within states to give us a wide range of diverse data points. Two datasets were utilized in the process. The first dataset consisted of 25 US states with statistics related to smoking, the most important being the rates at which smokers in the state attempted quitting (KFF, 2018). The second dataset reported COVID-19 death rates per state (Roser, Ritchie, Ortiz-Ospina, \& Joe Hasell, 2020). This gave us a direct correlation representing the relationship between nicotine withdrawal and COVID-19 mortality rates. While our study focuses on how cardiac arrhythmia caused by nicotine withdrawal affects COVID-19, we nevertheless wanted to prove a direct correlation between nicotine withdrawal and COVID-19, independent of heart diseases. However, due to due to the unavailability of datasets or clinical studies on specific patients who quit nicotine and then had COVID-19, we decided to find a trend across states.

\section{Results}

\section{Nicotine Withdrawal Symptoms}

From the clinical study on patients who had nicotine withdrawal, out of the 63 total patients involved in the study, we analyzed which individuals had at least one of the following: palpitations, various types of cardiac arrhythmia, strange heartbeat rhythms or intensities, and pounding heart. We found that 29 out of the 63 patients had either palpitations or cardiac arrhythmia, which equates to $46.03 \%$. 
Table 1. Summarized results from the NCBI clinical trial studying the outcomes of numerous patients after nicotine replacement therapy. Presented results include patient conditions before and after nicotine withdrawal.

\begin{tabular}{|c|c|c|c|c|}
\hline $\begin{array}{c}\text { \# of patients in } \\
\text { which quit nicotine }\end{array}$ & $\begin{array}{c}\text { \# of patients } \\
\text { previously healthy }\end{array}$ & $\begin{array}{c}\text { \# of patients with } \\
\text { palpitations/arrhythmia }\end{array}$ & $\begin{array}{c}\text { \# of patients with } \\
\text { pre-existing heart } \\
\text { conditions }\end{array}$ & Percentage (\%) \\
\hline 63 & 44 & 29 & 6 & 46.03 \\
\hline
\end{tabular}

We found that 14 patients saw palpitations, a symptom of cardiac arrhythmia, and 15 patients saw cardiac arrhythmia outright. Palpitations can develop into cardiac arrhythmia, so we wanted to include that probability in our final calculation. Thus, to find the chance of a patient seeing cardiac arrhythmia after smoking cessation, we had to weigh these numbers to calculate an average. After analyzing a study from the AAFP which followed 190 palpitation patients (Abbott, \& Allan V., 2005), it was found that 40\% of the patients developed cardiac arrhythmia.

We utilized a weighted average to calculate how often a patient who quits smoking will develop cardiac arrhythmia. The weighting factor is the probability that a patient will develop cardiac arrhythmia. Since different patients in the study have different probabilities of developing cardiac arrhythmia, using a weight for each patient would give the most accurate final probability. Since patients who have palpitations only develop arrhythmia $40 \%$ of the time, for those 14 patients, the weighting factor is 0.4 . For patients who immediately developed cardiac arrhythmia, their weighing factor is 1, since they have already developed it. Since 14/63 patients developed palpitations and $40 \%$ of patients who have palpitations eventually develop cardiac arrhythmia, there is an $8.9 \%$ chance they develop cardiac arrhythmia. Moreover, since 15/63 patients directly saw cardiac arrhythmia, their probability of developing cardiac arrhythmia is $23.8 \%$. Thus, by combining these rates, we got a final rate of $32.7 \%$, which is the probability that someone who quits smoking will develop cardiac arrhythmia.

\section{Equation 1:}

$$
x=\sum_{i=1}^{n}\left(x_{i} * w_{i}\right)
$$

Our equation is represented above, where $n=2, x_{1}=14 / 63, x_{2}=15 / 63, w_{1}=0.4, w_{2}=1$, and $x=32.7 \%$.

\section{Development Rates of Heart Complications}

To connect nicotine withdrawal with COVID-19, we first had to establish a relationship between COVID-19 and cardiac arrhythmia. A dataset from the CDC contained information regarding death counts for patients with COVID19 and a variety of other conditions, one being cardiac arrhythmia. By totaling the death counts for patients with both COVID-19 and cardiac arrhythmia across different states and age groups, we found that 6,932 patients had both COVID-19 and cardiac arrhythmia in the US as of July 17th. However, to calculate a final death rate of patients with cardiac arrhythmia and COVID-19, we were required to estimate the total cases of patients with both conditions, as no public datasets contained that information. Problematically, the dataset we utilized only recorded deaths, not total cases.

A compelling estimation strategy was to follow basic statistics and not consider any external factors, which inherently are unpredictable and easily unaccountable. The US cardiac arrhythmia rate is 5\% (Scripps Health, 2015), and this gave us a good baseline for estimation. We assumed that if 5\% of the US population has cardiac arrhythmia, and if every person has an equal chance of contracting COVID-19, then 5\% of COVID-19 patients will also have cardiac arrhythmia. We had to assume this because predicting who will get COVID-19 is impossible since COVID19 is not a disease someone develops, rather it is a disease that is contracted from exposure to other individuals. For example, COVID-19 isn't a condition which is developed, such as diabetes. Instead, it is more comparable to the 
common flu, which is contracted. Therefore, everyone should have the same chance of getting COVID-19. Since there still is little research on which demographics, let alone medical demographics, may be at higher or lower risk of getting COVID-19, this is the most effective way of making this estimation. For statistical proof, assume that the US population is subset A and the COVID-19 population is subset $\mathrm{B}$, and the cardiac arrhythmia population is subset C. Under the premise that COVID-19 randomly samples from the population, then subset B is a completely random subset of A. Since subset C is $5 \%$ of subset A, then by randomly sampling A, $5 \%$ of the subset B will contain members of subset C.

Based on the COVID-19 statistics as of July 17th, which is the last day that the CDC dataset recorded, there were 3,247,684 COVID-19 cases in the US (Roser, Ritchie, Ortiz-Ospina, \& Joe Hasell, 2020). Assuming 5\% of COVID-19 patients have cardiac arrhythmia, then 177,482 patients have both. As such, the death rate for patients with both cardiac arrhythmia and COVID-19 is approximately 3.9\%. The standard COVID-19 death rate for any person in the US is 3.4\% (Roser, Ritchie, Ortiz-Ospina, \& Joe Hasell, 2020), which means that for patients who have COVID19 and arrhythmia, the death rate increases by approximately 0.5 percentage points, or an increase of $14.7 \%$. Additionally, since the national death rate for patients with pre-existing cardiac arrhythmia is $0.96 \%$ (Scripps Health, 2015) and COVID-19 increases the death rate for these patients to 3.9\%, getting COVID-19 will increase their chance of death by $306 \%$.

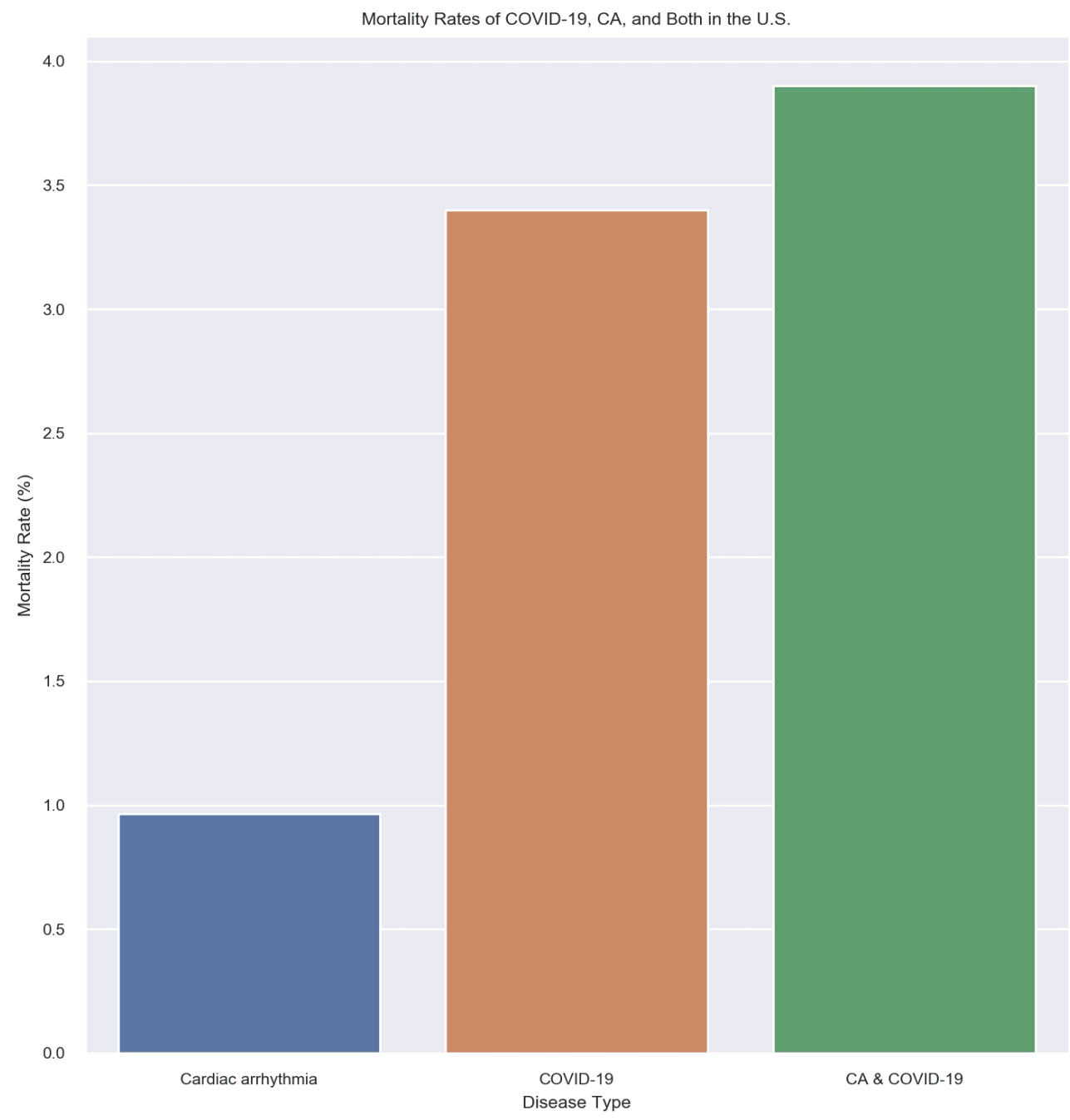

Figure 1. Mortality rates of cardiac arrhythmia and COVID-19 compared to the mortality rate of patients with both conditions (US Statistics). 


\section{Key Statistics}

We ultimately concluded that if a patient quits smoking, in the next 3 - 4 weeks, they have a $32.7 \%$ of developing cardiac arrhythmia, and if they then contract COVID-19, their chance of dying from COVID-19 will increase by $14.7 \%$ compared to a patient without cardiac arrhythmia. Thus, if a patient stops smoking, their chance of death from COVID-19 will increase by $4.8 \%$. This means for patients who quit smoking, their chance of death from COVID-19 is $3.56 \%$ due to the probability they develop cardiac arrhythmia.

Table 2. Relevant calculated results from our study. Death rates, increases in death rates, and chances of developing conditions are displayed.

\begin{tabular}{|c|c|c|c|c|}
\hline $\begin{array}{c}\text { Probability of } \\
\text { developing CA from } \\
\text { nicotine withdrawal } \\
(\%)\end{array}$ & $\begin{array}{c}\text { Mortality rate for } \\
\text { patients with CA }+ \\
\text { COVID-19 (\%) }\end{array}$ & $\begin{array}{c}\text { Increased mortality } \\
\text { rate of patients with } \\
\text { CA + COVID-19 vs } \\
\text { only COVID-19 (\%) }\end{array}$ & $\begin{array}{c}\text { Increased mortality } \\
\text { rate for CA patients } \\
\text { if they contract } \\
\text { COVID-19 (\%) }\end{array}$ & $\begin{array}{c}\text { Increased chance of } \\
\text { mortality from } \\
\text { COVID-19 } \\
\text { following nicotine } \\
\text { withdrawal (\%) }\end{array}$ \\
\hline 32.7 & 3.9 & 14.7 & 306 & 4.8 \\
\hline
\end{tabular}

\section{Validation Performance}

Finally, to prove a holistic trend between smoking cessation and COVID-19 death rates, we looked to different states to provide a wide range of data points. We analyzed two datasets, using one variable from each to support our validation. Our first variable was smoking cessation rates (KFF, 2018), and our second variable was COVID-19 death rates (Roser, Ritchie, Ortiz-Ospina, \& Joe Hasell, 2020). After isolating the 25 states provided in the first dataset, we visualized the relationship.

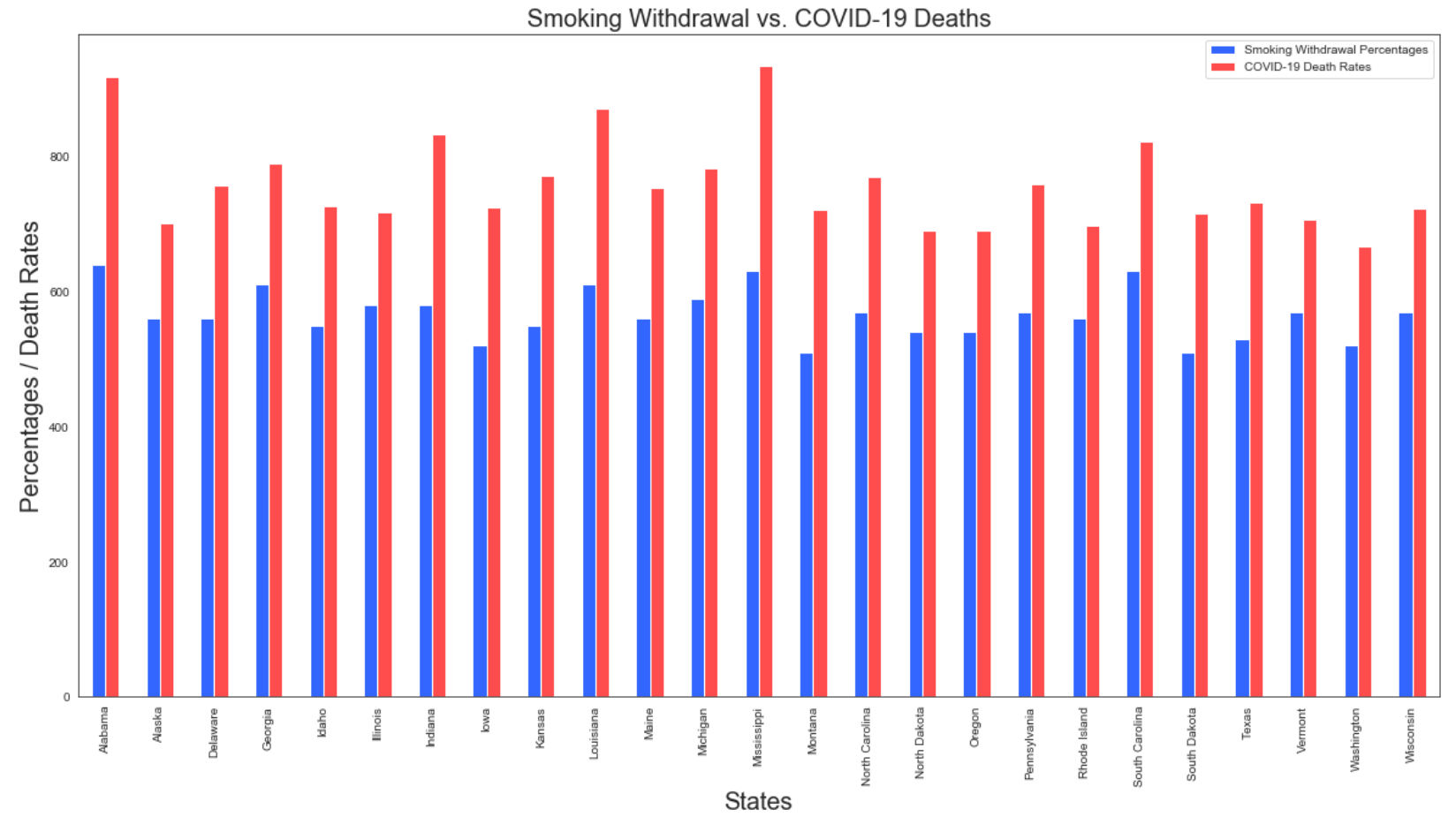

Figure 2. Smoking cessation rates against COVID-19 mortality rates in 25 US states provided in the dataset. Note: Cessation rates were scaled by a coefficient for visualization purposes. 
After seeing probable correlation, we proceeded to create a linear regression model to analyze the correlation in-depth. We expected to see a positive correlation between the rates of smoking cessation and COVID-19 death rates.

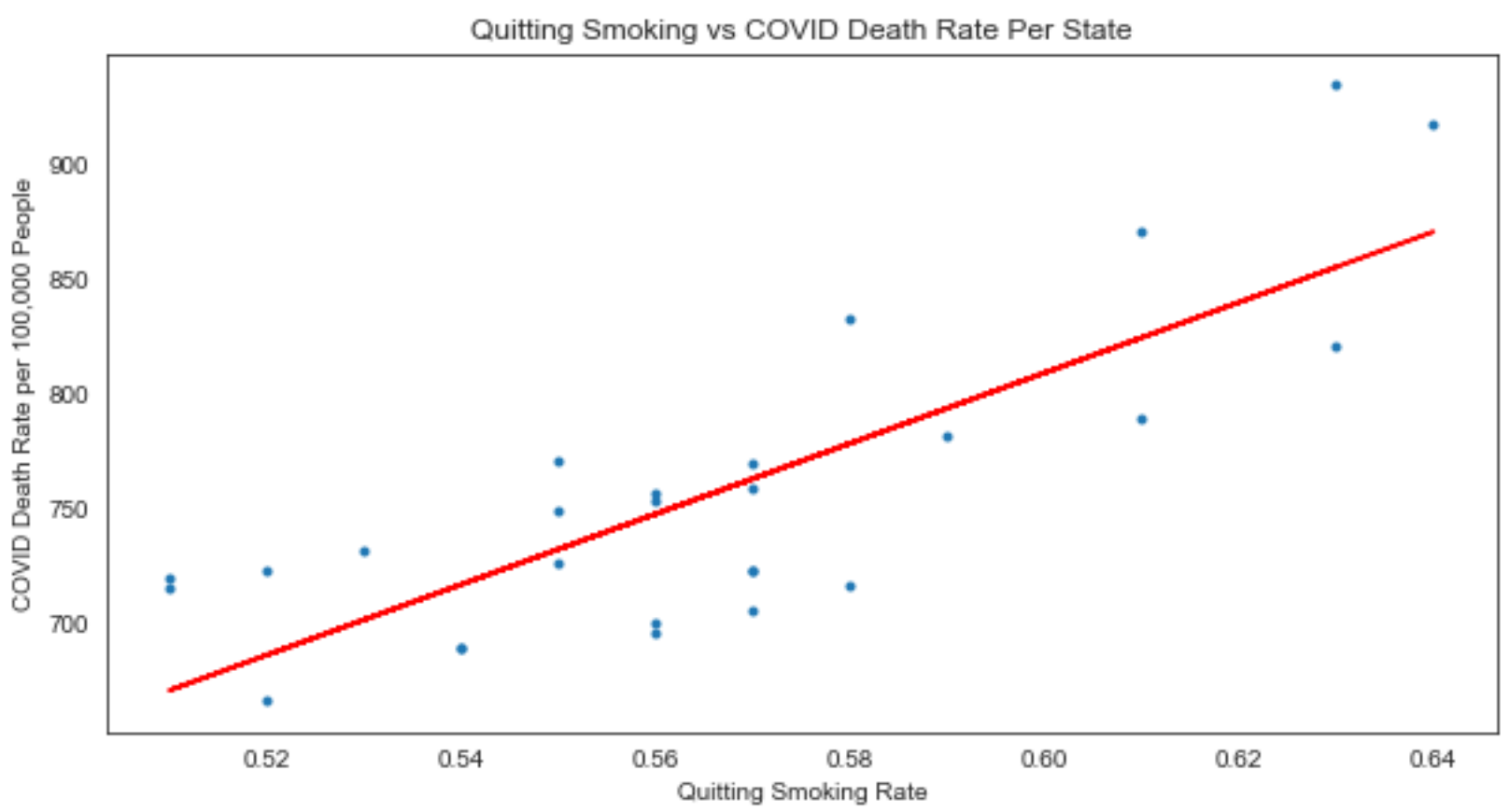

Figure 3. Regression model representing the correlation between smoking cessation rates and COVID-19 death rates for 25 US states with recorded data.

The regression line had a correlation factor of 0.805 . The relationship between two variables is generally considered strong when their correlation coefficient is larger than 0.7 (Mindrila, 2013). Hence, our calculations and conclusions proved to be accurate and realistic. Each condition in our study was proven to be carefully connected with each other condition, as nicotine withdrawal has a direct connection with both cardiac arrhythmia and COVID-19. Without this validation study, our results would have been solely contingent on cardiac arrhythmia.

\section{Discussion}

Our results could potentially help active smokers by informing them of the risks of nicotine withdrawal. Patients with nicotine addictions could benefit from our findings by understanding how nicotine withdrawal will increase COVID19 mortality rates.

Although numerous studies claim that smokers should attempt to quit in order to decrease their risk of death from COVID-19, our research is still very beneficial. We have found nicotine withdrawal increases the COVID-19 mortality rates by $4.8 \%$ in the first 3 - 4 weeks after withdrawal. However, an observational database from 169 hospitals in Asia, Europe, and North America, found that an active smoker is 1.8 times more likely to die than a normal, healthy patient with no underlying health conditions (Mehra, Desai, Kuy, Henry, \& Patel, 2020). We recognize that smoking does lead to a higher death rate from COVID-19, but we also prove the fact that deliberate withdrawal could have adverse effects. While it seems clear that quitting smoking is a preferable action to take, we do believe that our research is important to patients, as they can be aware of the potential side effects of nicotine withdrawal and how that may play a role in the outcomes of COVID-19. It is crucial to present both sides of an action as our research has 
proven that nicotine withdrawal has its own definite harms. Rather than immediately quitting smoking, smokers could follow a more gradual process for smoking cessation in order to lower their chance of death from COVID-19.

A shortcoming of our research lies in the inherent problem of estimating values. While we prove our estimations are statistically sound and mathematically probable, estimations are still inherently guesses and have the propensity to be incorrect. Since lots of data related to COVID-19 is either not collected or not publicly available, we were forced to make estimations. Our estimations may have been over or underestimated, but the deviation of our estimations from ground-truth values should not be statistically significant. When this data is released in the future, we can review our estimations and present more accurate findings.

Lastly, nicotine withdrawal does not only pertain to a complete halt to nicotine consumption, as simply reducing the amount of nicotine consumed can lead to withdrawal side effects. For example, when patients attempt to quit smoking, they may use nicotine patches or other alternatives which still contain nicotine. Future studies, contingent on datasets and trials, could draw a correlation between the amount of nicotine decreased and the chance of developing cardiac arrhythmia and the COVID-19 mortality rates.

\section{Conclusion}

Nicotine withdrawal has been shown to have negative side effects, and through our research, we prove that these side effects lead to the development of cardiac arrhythmia, which increases the chance of death from COVID-19. During the pandemic, it has generally been advised that quitting smoking is an effective way to reduce the negative effects of COVID-19, but the side effects of nicotine withdrawal and how they may affect COVID-19 mortality rates are often unconsidered. Through the use of computational and statistical analysis, we found that if a patient quits smoking, they have a $32.7 \%$ chance of developing cardiac arrhythmia. Cardiac arrhythmia was found to increase the COVID-19 mortality rate by $14.7 \%$, and COVID-19 was found to increase the death rate of cardiac arrhythmia by $306 \%$. Thus, for patients who quit smoking, if they contract COVID-19, their mortality rate increases $4.8 \%$. The potential negatives of nicotine withdrawal on COVID-19 are important for health officials and patients to understand, as it can help patients develop a gradual approach to smoking cessation. Future studies could draw correlations regarding how other symptoms of nicotine withdrawal could also increase COVID-19 mortality rates, which could develop into even more serious health and life-threatening complications.

\section{Acknowledgments}

We would like to thank Keshav Rao, our advisor, for guiding us through this research project. We would also like to thank ASDRP, Fremont STEM, and the Olive Children Foundation for making this research possible and providing us with the resources. All authors had similar contributions in the research process and paper writing. Our source code can be found here: https://github.com/ayaanzhaque/Nicotine-COVID19

\section{Abbreviations}

CA, Cardiac Arrhythmia

CDC, Centers for Disease Control and Prevention

HHS, The United States Department of Health and Human Services

NCBI, National Center for Biotechnology Information

WHO, World Health Organization 


\section{References}

Abbott, A. (2005, February 15). Diagnostic Approach to Palpitations. Retrieved September 14, 2020, from https://www.aafp.org/afp/2005/0215/p743.html

Antzelevitch, C. (2007). Heterogeneity and cardiac arrhythmias: An overview. Heart Rhythm, 4(7), 964-972. doi:10.1016/j.hrthm.2007.03.036

Brugada, P., Andries, E., Gürsoy, S., \& Brugada, J. (1993). Investigation of palpitations. The Lancet, 341(8855), 1254-1258. doi:10.1016/0140-6736(93)91155-f

Center for Disease Control and Prevention. (2019, November 18). Current Cigarette Smoking Among Adults in the United States. Retrieved September 14, 2020, from https://www.cdc.gov/tobacco/data statis$\underline{\text { tics/fact sheets/adult data/cig smoking/index.htm }}$

Department of Health \& Human Services. (2013, April 30). Heart arrhythmias and palpitations. Retrieved September 14, 2020, from https://www.betterhealth.vic.gov.au/health/conditionsandtreatments/heart-arrhythmias-and-palpi$\underline{\text { tations }}$

Kaiser Family Foundation. (2020, January 03). Smokers Who Report Attempting to Quit Smoking by Race/Ethnicity. Retrieved September 14, 2020, from https://www.kff.org/other/state-indicator/attempts-to-quit-smoking

Mehra, M., Al., E., Author AffiliationsFrom Brigham and Women's Hospital Heart and Vascular Center and Harvard Medical School, Others, J., Rubin, E., Others, C., . . Ehre, C. (2020, June 18). Cardiovascular Disease, Drug Therapy, and Mortality in Covid-19: NEJM. Retrieved September 14, 2020, from https://www.nejm.org/doi/full/10.1056/NEJMoa2007621

Mills, E. J., Thorlund, K., Eapen, S., Wu, P., \& Prochaska, J. J. (1970, January 01). Cardiovascular events associated with smoking cessation pharmacotherapies: A network meta-analysis. Retrieved September 14, 2020, from https://www.ncbi.nlm.nih.gov/pubmedhealth/PMH0062030/

Mindrila, D., Balentyne P.. (2013). Scatterplots and Correlation. Retrieved from https://www.westga.edu/academics/research/vrc/assets/docs/scatterplots and correlation notes.pdf

Mjøs, O. D. (1988). Lipid effects of smoking. American Heart Journal, 115(1), 272-275. doi:10.1016/00028703(88)90649-7

National Center for Health Statistics. (2020). Conditions contributing to deaths involving coronavirus ... Retrieved September 14, 2020, from https://data.cdc.gov/NCHS/Conditions-contributing-to-deaths-involving-corona/hk9yquqm

Redaction, U. (2020, July 18). Number of COVID-19 cases confirmed worldwide exceeds 14.1 mln. Retrieved September 14, 2020, from https:/www.unian.info/world/covid-19-worldwide-number-of-cases-confirmed-worldwideexceeds-14-1-mln-11079707.html

Roser, M., Ritchie, H., Ortiz-Ospina, E., \& Hasell, J. (2020, March 04). Coronavirus Pandemic (COVID-19) - Statistics and Research. Retrieved September 14, 2020, from https://ourworldindata.org/coronavirus 
Scripps Health SD. (2015). Top 10 Things You Should Know About Heart Rhythm. Retrieved September 14, 2020, from https://www.scripps.org/sparkle-assets/documents/heart rhythm facts.pdf

Wadgave, U., \& L., N. (2016). Nicotine Replacement Therapy: An Overview. International Journal of Health Sciences, 10(3), 407-416. doi:10.12816/0048737 